\title{
Ordinal products of topological spaces
}

\author{
by
}

\author{
V. A. Chat yrko (Moscow)
}

\begin{abstract}
The notion of the ordinal product of a transfinite sequence of topological spaces which is an extension of the finite product operation is introduced. The dimensions of finite and infinite ordinal products are estimated. In particular, the dimensions of ordinary products of Smirnov's [S] and Henderson's [He1] compacta are calculated.
\end{abstract}

Introduction. The necessary information about the notions and notations we use can be found in [A-Pa], [E1], [E2], [K-M] and in the appendix.

One of the main questions in transfinite dimension theory is

The Problem of PRoduct Dimension (PPD). Let DIM be a transfinite dimension function, for example: ind, Ind, $\operatorname{dim}_{\mathrm{w}}, \operatorname{dim}_{\mathrm{c}}, D$, and suppose $U$ is a fixed class of topological spaces. What can be said about the dimension DIM of the product of two spaces $X, Y$ from the class $U$ if this dimension is defined for the factors?

Let us give some possible concretizations of (PPD):

(1) Does DIM $X \times Y$ exist?

(2) Is there an (optimal) transfinite function $\Phi=\Phi(\alpha, \beta)$ of two transfinite variables such that

$$
\operatorname{DIM} X \times Y \leq \Phi(\operatorname{DIM} X, \operatorname{DIM} Y) ?
$$

(The function $\Phi(\alpha, \beta)$ is called optimal if for every pair of transfinite numbers $\alpha, \beta$ there are spaces $X=X(\alpha, \beta)$ and $Y=Y(\alpha, \beta)$ in $U$ such that DIM $X=$ $\alpha, \operatorname{DIM} Y=\beta$, and $\operatorname{DIM} X \times Y=\Phi(\alpha, \beta)$.)

(3) What is the value of DIM $X \times Y$ ?

In this paper we will be interested in questions (2), (3) and their generalizations. In the introduction we discuss the case of metric compacta unless otherwise stated.

1991 Mathematics Subject Classification: Primary 54F45. 
For the traditional transfinite dimensions ind and Ind (see $[\mathrm{A}-\mathrm{Pa}]$ ) the inequality

$$
\operatorname{DIM} X \times Y<\omega_{1}
$$

is well known, and it is equivalent to the existence of DIM $X \times Y$ for these dimensions. The analogous statement is true for $\operatorname{dim}_{\mathrm{c}}$, the transfinite extension of the Lebesgue covering dimension $\operatorname{dim}$ to compact $C$-spaces ([B3] and $[\mathrm{Ha}-\mathrm{Y}])$. A more delicate result for ind has been obtained by Toulmin $[\mathrm{T}]$ : ind $X \times Y \leq($ ind $X(+)$ ind $Y)+n$, where $n$ is a finite non-negative integer which depends on the inductive dimensions of $X$ and $Y$, and $(+)$ is the natural sum of Hessenberg [Hes].

For any metrizable compactum $Z$ we have Ind $Z \leq \omega \cdot$ ind $Z$ [Le], which leads to a more precise estimate for Ind than $(*)$. Note that an improvement of $(*)$ for Ind for a certain class of topological spaces has also been stated by Polkowski $[\mathrm{Po}]$. For the dimension $D$ the inequality $D(X \times Y) \leq D X(+) D Y$ has been proved by Henderson [He2].

So $\operatorname{PPD}(2)$ for the dimensions indicated above reduces either to obtaining an optimal estimating function $\Phi$ or to the proof of optimality of a given one. Note that for $\operatorname{dim}_{\mathrm{w}}$, another transfinite extension of dim to weakly infinite-dimensional compacta [B1], even the rough estimate $(*)$ has not been obtained yet. This is the well-known problem of the weak infinite-dimensionality of the product of weakly infinite-dimensional compacta. Note that $\operatorname{PPD}(2)$ coincides with $\operatorname{PPD}(3)$ in the part which deals with optimality - one has to calculate the dimension of the product of the chosen pair of compacta. As far as I know the calculation of the dimension of the product of two infinite-dimensional compacta has not been made yet.

In [S] Smirnov constructed compacta $S^{\alpha}$ with Ind $S^{\alpha}=\alpha, \alpha<\omega_{1}$, and from this he deduced that there are no universal spaces in the class of countable-dimensional metric compacta. Smirnov's construction turned out to be very useful. Using its modification Henderson [He1] constructed AR-compacta $H^{\alpha} \hookleftarrow S^{\alpha}$ with Ind $H^{\alpha}=\alpha, \alpha<\omega_{1}$, and defined for them the notion of an essential mapping. He also proved that $D S^{\alpha}=\alpha$ [He2]. In [B1], [B2] Borst, having extended the covering dimension dim to ordinals, proved a transfinite analog of Aleksandrov's theorem on essential mappings for locally compact metric spaces, namely: $\operatorname{dim}_{\mathrm{w}} X \geq \alpha$ iff $X \times C$ has an essential mapping onto $H^{\alpha}$, where $C$ is the Cantor middle thirds set $\left(\operatorname{dim}_{\mathrm{w}}\right.$ is the above mentioned transfinite extension of dim). He also proved the equalities $\operatorname{dim}_{\mathrm{w}} S^{\alpha}=\operatorname{dim}_{\mathrm{w}} H^{\alpha}=\alpha$ for $\alpha<\omega_{1}$, from which one sees directly that there is no universal space in the class of weakly infinite-dimensional metric compacta (the weak infinite-dimensionality of a metric compactum $X$ is equivalent to the inequality $\operatorname{dim}_{\mathrm{w}} X<\omega_{1}$ [B1]). Note that the non-existence of universal spaces in the class of weakly infinite-dimensional compacta also 
follows from an earlier result of Pol $[\mathrm{P}]$ connected with Smirnov compacta. Namely:

If a complete space $X$ topologically contains every Smirnov compactum $S^{\alpha}$, then $X$ topologically contains the Hilbert cube. Hence $X$ is not weakly infinite-dimensional.

Naturally PPD(3) arises for useful and easily constructed Smirnov and Henderson compacta. In this paper this question is completely solved. It turns out that

$$
\operatorname{DIM} X^{\alpha} \times X^{\beta}=\alpha(+) \beta,
$$

where DIM is Ind, Id (to be defined below), $\operatorname{dim}_{\mathrm{w}}$, or $D$ and $X^{\gamma}, \gamma<\omega_{1}$, are either the Smirnov compacta $S^{\gamma}$ or the Henderson compacta $H^{\gamma}$.

The paper consists of three parts. In the first part, starting from Smirnov's construction we suggest the definition of an infinite product of topological spaces - the ordinal $\aleph_{0}$-product (Definition 1) for which, in contrast to Tikhonov products, there are non-trivial solutions of the natural extension of PPD to an infinite number of non-zero-dimensional factors (Theorem 4).

Let, for example, $S=\left\{X_{\gamma}, \gamma<\beta\right\}$ be a set of compacta indexed by ordinals $<\beta$ (such sets will be called $\beta$-sequences). Then the compactum

$$
\prod_{\gamma<\beta}^{\omega, \text { ord }} X_{\gamma}= \begin{cases}\left(\prod_{\gamma<\beta-1}^{\text {point }} X_{\gamma}\right) \times X_{\beta-1} & \text { if } \beta \text { is a non-limit ordinal } \\
\text { Aleksandrov compactification of the free sum } \\
\left(\begin{array}{l}
(+) \\
\delta<\beta
\end{array}\left(\prod_{\gamma<\delta}^{\omega, \text { ord }} X_{\gamma}\right)\right) \times \mathbb{N} & \text { if } \beta \text { is a limit ordinal, where } \\
& \mathbb{N} \text { are the natural numbers }\end{cases}
$$

is the ordinal $\aleph_{0}$-product of the $\beta$-sequence $S$. If all the $X_{\gamma}$ are homeomorphic to $X$, then $\prod_{\gamma<\beta}^{\omega, \text { ord }} X_{\gamma}$ is called the $\beta$-ordinal $\aleph_{0}$-power and is denoted by $S_{\beta}^{\omega}(X)$. In particular, if $\beta<\omega_{1}$ and $I$ is the interval $[0,1]$ then $S^{\beta} \hookrightarrow S_{\beta}^{\omega}(I) \hookrightarrow S^{\beta}$, where $S^{\beta}$ is the Smirnov compactum $(\hookrightarrow$ denotes closed embedding).

The new product, just as the Tikhonov product, is an extension of the notion of a finite topological product to an infinite number of factors, but in contrast to the latter, it essentially depends on the order of the indexed set of factors. For example, for two different countable ordinals $\alpha$ and $\beta$ the $\alpha$ - and $\beta$-ordinal $\aleph_{0}$-powers of the interval are not homeomorphic because Ind $S^{\alpha}=\alpha \neq \beta=\operatorname{Ind} S^{\beta}$. Let us state one of the main results of the paper which explains why $\aleph_{0}$-products are called products.

TheOREM 1. Let $X$ be an arbitrary topological space and $\alpha, \beta$ be countable ordinals. Then

$$
S_{\alpha}^{\omega}(X) \times S_{\beta}^{\omega}(X)=S_{\alpha(+) \beta}^{\omega}(X)
$$


From Theorem 1 which reminds the main property of the power, one directly obtains

COROllary 2. Let $\Phi$ be a numerical function on topological spaces, monotone on closed subsets, for example a dimension (ind, Ind, $\operatorname{dim}_{\mathrm{w}}, D$ or others). Then

$$
\Phi\left(S_{\alpha}^{\omega}(X) \times S_{\beta}^{\omega}(X)\right)=\Phi\left(S_{\alpha(+) \beta}^{\omega}(X)\right) .
$$

In particular, for Smirnov compacta one has:

(a) DIM $S^{\alpha} \times S^{\beta}=\alpha(+) \beta$, where DIM is $\operatorname{dim}_{\mathrm{w}}$, Ind, Id or $D$;

(b) ind $S^{\alpha} \times S^{\beta}=$ ind $S^{\alpha(+) \beta}$;

(c) $S^{\alpha} \times S^{\beta}$ can be continuously mapped into $[0,1]$ so that every point of the interval has a finite-dimensional preimage.

One can easily see that an upper estimate of the dimension of $S^{\alpha} \times S^{\beta}$ is obtained from the inclusion $S^{\alpha} \times S^{\beta} \hookrightarrow S^{\alpha(+) \beta}$, which is not true for Henderson compacta. For these, the necessary estimate is deduced from the second part of the paper, where for the transfinite extension of the finite dimension Id, introduced by Pasynkov [Pa1], we give the optimal solution of $\operatorname{PPD}(2)$, namely:

Let $X, Y$ be compacta for which $\operatorname{Id}$ is defined. Then $\operatorname{Id} X \times Y \leq$ $\operatorname{Id} X(+) \operatorname{Id} Y$.

This inequality, obtained as a corollary of the more general Theorem 3, makes it possible to give an optimal upper bound for the dimensions Ind, $\operatorname{dim}_{\mathrm{w}}$ of products of compacta under natural additional assumptions (Corollary 6). Note that this inequality has been independently obtained by Vinogradov. Also note that the obtained estimate for Id, just as Henderson's inequality for $D$, is optimal (use Smirnov compacta).

In the third part questions connected with the dimension of infinite ordinal products are discussed. In particular, we prove the following transfinite generalization to ordinal $\aleph_{0}$-products of the Brower theorem on the $n$-dimensionality of the cube $I^{n}$.

Theorem 5. Let DIM be Ind, Id or $\operatorname{dim}_{\mathrm{w}}$, and let $X_{\gamma}, \gamma<\beta$, be onedimensional metric compacta. Then

$$
\operatorname{DIM} \prod_{\gamma<\beta}^{\omega, \text { ord }} X_{\gamma}=\beta .
$$

1. Ordinal products of topological spaces (a special case). Let $X_{\alpha}, \alpha \in A,|A| \geq \aleph_{0}$, be a family of topological spaces. The one-point Aleksandrov extension of the free sum of the spaces $X_{\alpha}, \alpha \in A$, is the space $X=\{*\} \cup(+)_{\alpha \in A} X_{\alpha}$, formed from $(+)_{\alpha \in A} X_{\alpha}$ by adding the point $*$ with the following topology: 
- $X_{\alpha}$ is clopen in $X$ for all $\alpha \in A$;

- the sets $X \backslash\left\{X_{\alpha_{1}}(+) \ldots(+) X_{\alpha_{k}}\right\}, \alpha_{i} \in A, i=1, \ldots, k, k \in \mathbb{N}$, generate the base of the topology at $*$.

Obviously, if all $X_{\alpha}, \alpha \in A$, are compact, then the one-point Aleksandrov extension coincides with the one-point Aleksandrov compactification.

Let us list some elementary properties of the one-point Aleksandrov extension. The notation $Z \simeq Y$ will mean that the spaces $Z$ and $Y$ are homeomorphic. Let $X=\{*\} \cup(+)_{\alpha \in A} X_{\alpha}$. Then

- if $V$ is an open subset of $X \backslash\{*\}$ such that $B=\left\{\alpha \in A:(X \backslash V) \cap X_{\alpha}\right.$ $\neq \emptyset\}$ has cardinality $\geq \aleph_{0}$, then $X \backslash V=\{*\} \cup(+)_{\alpha \in B}\left(X_{\alpha} \backslash V\right) \hookrightarrow X$;

- if $Y_{\alpha} \hookrightarrow X_{\alpha}$ for all $\alpha \in A$, then $\{*\} \cup(+)_{\alpha \in A} Y_{\alpha} \hookrightarrow X$;

- if for every $\alpha \in A$ the space $X_{\alpha}$ is Hausdorff (Tikhonov, pseudocompact, normal, paracompact, compact, $S$-weakly infinite-dimensional, a $C$-space), then so is $X$;

- if $|A|=\aleph_{0}$ and for every $\alpha \in A$ the space $X_{\alpha}$ is perfectly normal (metrizable, with a countable base), then so is $X$;

- $\boldsymbol{\beta}\left(\{*\} \cup(+)_{\alpha \in A} X_{\alpha}\right)=\{*\} \cup(+)_{\alpha \in A} \boldsymbol{\beta} X_{\alpha}$ where $\boldsymbol{\beta}$ denotes the CechStone compactification.

A family $S=\left\{X_{\gamma}, \gamma<\beta\right\}$ of topological spaces indexed by ordinals $<\beta$ will be called a $\beta$-sequence of topological spaces.

Definition 1. The ordinal product (resp. ordinal $\aleph_{0}$-product) of a $\beta$ sequence $S=\left\{X_{\gamma}, \gamma<\beta\right\}$ of topological spaces is, respectively, the topological space

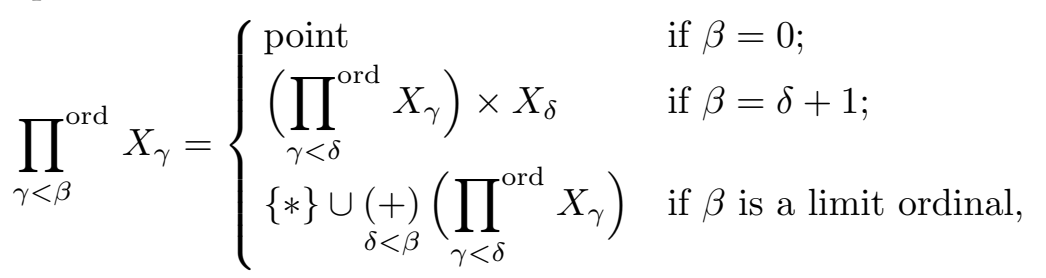

and

$$
\prod_{\gamma<\beta}^{\omega, \text { ord }} X_{\gamma}= \begin{cases}\left.\prod_{\gamma<\delta}^{\text {point }} X_{\gamma}\right) \times X_{\delta} \text { ord } & \text { if } \beta=0 \\ \{*\} \cup(+)\left\{\left(\prod_{\gamma<\delta}^{\omega, \text { ord }} X_{\gamma}\right)_{i}: \delta<\beta, i<\omega\right\} & \text { if } \beta=\delta+1\end{cases}
$$

Here $\left(\prod_{\gamma<\delta}^{\omega, \text { ord }} X_{\gamma}\right)_{i} \simeq \prod_{\gamma<\delta}^{\omega, \text { ord }} X_{\gamma}, i<\omega$. The notation $\prod_{\gamma<\delta}^{(\omega) \text {,ord }} X_{\gamma}$ will mean either $\prod_{\gamma<\delta}^{\omega, \text { ord }} X_{\gamma}$ or $\prod_{\gamma<\delta}^{\text {ord }} X_{\gamma}$.

Let us list some elementary properties of ordinal products. Let $X=$ $\prod_{\gamma<\beta}^{(\omega), \text { ord }} X_{\gamma}$. Then 
- if $Y_{\gamma} \hookrightarrow X_{\gamma}$ for every $\gamma<\beta$, then $\prod_{\gamma<\beta}^{(\omega), \text { ord }} Y_{\gamma} \hookrightarrow X$;

- if $\delta<\beta$, then $\prod_{\gamma<\delta}^{(\omega) \text {,ord }} X_{\gamma} \hookrightarrow X$;

- if for every $\gamma<\beta$ the space $X_{\gamma}$ is a Hausdorff (Tikhonov, compact, compact $C$-) space, then so is $X$;

- if $\beta<\omega_{1}$ and for every $\gamma<\beta$ the space $X_{\gamma}$ is metrizable (with a countable base), then so is $X$;

- if $X$ is pseudocompact, then

$$
\boldsymbol{\beta} X=\prod_{\gamma<\beta}^{(\omega), \text { ord }} \boldsymbol{\beta} X_{\gamma} .
$$

Recall that the product of two compact $C$-spaces is a compact $C$-space [Ha-Y], and if $X \times Y$ is pseudocompact, then $\boldsymbol{\beta}(X \times Y)=\boldsymbol{\beta} X \times \boldsymbol{\beta} Y$ [E1].

2. Ordinal power of a topological space (a special case). Let $X$ be a topological space and $S=\left\{X_{\gamma}, \gamma<\beta\right\}$ be a $\beta$-sequence of topological spaces such that $X_{\gamma} \simeq X$ for every $\gamma<\beta$.

Definition 2. The $\beta$-ordinal power (resp. $\aleph_{0}$-power) of $X$ is the space $S_{\beta}(X)=\prod_{\gamma<\beta}^{\text {ord }} X_{\gamma}\left(\operatorname{resp} . S_{\beta}^{\omega}(X)=\prod_{\gamma<\beta}^{\omega, \text { ord }} X_{\gamma}\right)$.

The notation $S_{\beta}^{(\omega)}(X)$ will mean either $S_{\beta}^{\omega}(X)$ or $S_{\beta}(X)$.

It is clear that, if $1 \leq \beta<\omega_{1}$, then

- $S_{\beta}(I)$ is the Smirnov compactum $S^{\beta}$;

- $C \hookrightarrow S_{\beta}(C) \hookrightarrow C$, where $C$ is the Cantor set.

Let us state some elementary properties of ordinal powers:

- if $X \hookrightarrow Y$, then $S_{\beta}^{(\omega)}(X) \hookrightarrow S_{\beta}^{(\omega)}(Y)$;

- if $\beta<\alpha$, then $S_{\beta}^{(\omega)}(X) \hookrightarrow S_{\alpha}^{(\omega)}(X)$.

The following statement will often be used below.

Lemma 1. Let $\alpha<\omega_{1}$. Then

(a) if $\left\{\alpha_{i}\right\}_{i=1}^{\infty}$ is a sequence of ordinals such that $\alpha_{i}<\alpha_{i+1}$ and $\sup _{i} \alpha_{i}=$ $\alpha$, then

$$
S_{\alpha}^{(\omega)}(X) \hookrightarrow\{*\} \cup \underset{i=1}{\stackrel{\infty}{\infty})} S_{\alpha_{i}}^{(\omega)}(X) \hookrightarrow S_{\alpha}^{(\omega)}(X) ;
$$

(b) $S_{\alpha}(X) \hookrightarrow S_{\alpha}^{\omega}(X) \hookrightarrow S_{\alpha}(X)$;

(c) if $\alpha$ is a limit ordinal, then $S_{\alpha}^{\omega}(X) \simeq S_{\alpha}^{\omega}(X) \backslash\{$ a finite number of terms of the free sum defining $\left.S_{\alpha}^{\omega}(X)\right\}$.

The proof is obvious.

We now prove the finite multiplicativity of $\aleph_{0}$-powers for countable ordinals. 
Theorem 1. Let $\alpha, \beta<\omega_{1}$. Then

$$
S_{\alpha}^{\omega}(X) \times S_{\beta}^{\omega}(X)=S_{\alpha(+) \beta}^{\omega}(X)
$$

(here $\alpha(+) \beta$ is the natural sum of the ordinals $\alpha$ and $\beta$; see appendix).

Pro of. We use induction. Let $\beta=1$, and let $\alpha$ be an ordinal $<\omega_{1}$. By the definition we have

$$
S_{\alpha}^{\omega}(X) \times X=S_{\alpha(+) 1}^{\omega}(X) .
$$

Assume that for $\beta<\nu$ and for all $\alpha<\omega_{1}$ our statement is true, and let $\beta=\nu$. Suppose $\beta=\varepsilon+1$. Then by the definition and the inductive assumption one can easily check that

$$
\begin{aligned}
S_{\alpha}^{\omega}(X) \times S_{\beta}^{\omega}(X) & =S_{\alpha}^{\omega}(X) \times S_{\varepsilon}^{\omega}(X) \times X \\
& =S_{\alpha+1}^{\omega}(X) \times S_{\varepsilon}^{\omega}(X)=S_{(\alpha+1)(+) \varepsilon}^{\omega}(X)=S_{\alpha(+) \beta}^{\omega}(X) .
\end{aligned}
$$

Let now $\beta$ be a limit ordinal. We now use induction on $\alpha$. For $\alpha=1$ the statement is obvious. Suppose that for every $\alpha<\mu$ and the fixed limit $\beta$ the statement is true, and let $\alpha=\mu$. Assume that $\alpha=\varepsilon+1$. Then by the definition and the inductive assumption we have

$$
S_{\alpha}^{\omega}(X) \times S_{\beta}^{\omega}(X)=S_{\varepsilon}^{\omega}(X) \times S_{\beta}^{\omega}(X) \times X=S_{\varepsilon(+) \beta}^{\omega}(X) \times X=S_{\alpha(+) \beta}^{\omega}(X) .
$$

Let now $\alpha$ be a limit ordinal. By Definition 1 one has

$$
S_{\alpha}^{\omega}(X)=\left\{*_{1}\right\} \cup(+)\left\{\left(S_{\delta}^{\omega}(X)\right)_{i}: \delta<\alpha, i<\omega\right\} .
$$

Note that for a fixed $\delta<\alpha$ the space $S_{\delta}^{\omega}(X)$ appears in the free sum countably many times. Let us number all spaces of the free sum by positive integers:

$$
S_{\alpha}^{\omega}(X)=\left\{*_{1}\right\} \cup \underset{i=1}{\stackrel{\infty}{+})} X_{i},
$$

and the same for $S_{\beta}^{\omega}(X)$ :

$$
S_{\beta}^{\omega}(X)=\left\{*_{2}\right\} \cup \underset{i=1}{\stackrel{\infty}{+})} Y_{i} .
$$

We also number by positive integers all different ordinals of the form $\delta(+) \eta$, where $\delta<\alpha$ and $\eta<\beta$ :

$$
\{\delta(+) \eta: \delta<\alpha, \eta<\beta\}=\left\{\gamma_{1}, \gamma_{2}, \ldots\right\} .
$$

By the inductive assumption the product $X_{k} \times Y_{l}, k, l<\omega$, is homeomorphic to $S_{\xi}^{\omega}$, where $\xi=\delta(+) \eta$ for some $\delta<\alpha$ and $\eta<\beta$, and $X_{k}=S_{\delta}^{\omega}(X)$, $Y_{l}=S_{\eta}^{\omega}(X)$. Consider an increasing sequence $\{m(i)\}_{i=0}^{\infty}$ of positive integers with $m(0)=1$ such that for every $i<\omega$ the spaces $S_{\gamma_{j}}(X), j=1, \ldots, i$, occur in the free sum

$$
(+)\left\{X_{k} \times Y_{l}: m(i) \leq k, l<m(i+1)\right\} .
$$


Hence in the free sum

$$
(+)\left\{X_{k} \times Y_{l}: m(i) \leq k, l<m(i+1), i<\omega\right\}
$$

there are countably many spaces $S_{\gamma_{j}}(X)$, for $j=1,2, \ldots$ Clearly,

$$
\begin{aligned}
& S_{\alpha}^{\omega}(X) \times S_{\beta}^{\omega}(X)=\{*\} \cup(+)\{ X_{k} \times Y_{l} \\
&(+) X_{k} \times\left(S_{\beta}^{\omega}(X) \backslash(+)\left\{Y_{p}: p<m(i+1)\right\}\right) \\
&(+)\left(S_{\alpha}^{\omega}(X) \backslash(+)\left\{X_{p}: p<m(i+1)\right\}\right) \times Y_{l}: \\
&m(i) \leq k, l<m(i+1), i<\omega\} .
\end{aligned}
$$

By Lemma 1(c) for every $i<\omega$ one has

$$
\begin{aligned}
S_{\alpha}^{\omega}(X) \backslash(+)\left\{X_{p}: p<m(i+1)\right\} & =S_{\alpha}^{\omega}(X), \\
S_{\beta}^{\omega}(X) \backslash(+)\left\{Y_{p}: p<m(i+1)\right\} & =S_{\beta}^{\omega}(X) .
\end{aligned}
$$

Hence

$$
\begin{aligned}
S_{\alpha}^{\omega}(X) \times & S_{\beta}^{\omega}(X) \\
=\{*\} \cup(+)\left\{X_{k} \times Y_{l}(+) X_{k} \times S_{\beta}^{\omega}(X)(+) S_{\alpha}^{\omega}(X) \times Y_{l}:\right. & m(i) \leq k, l<m(i+1), i<\omega\} .
\end{aligned}
$$

Recall that $X_{k}=S_{\delta}^{\omega}(X)$ and $Y_{l}=S_{\eta}^{\omega}(X)$ for some $\delta<\alpha$ and $\eta<\beta$, $k, l<\omega$. Hence by the inductive assumption one has

$$
\begin{aligned}
X_{k} \times S_{\beta}^{\omega}(X) & =S_{\delta}^{\omega}(X) \times S_{\beta}^{\omega}(X)=S_{\delta(+) \beta}^{\omega}(X), \\
S_{\alpha}^{\omega}(X) \times Y_{l} & =S_{\alpha}^{\omega}(X) \times S_{\eta}^{\omega}(X)=S_{\alpha(+) \eta}^{\omega}(X) .
\end{aligned}
$$

Moreover, by Lemma A1 of the appendix for every $\gamma<\alpha(+) \beta$ there exist ordinals $\alpha_{1}, \beta_{1}$ such that $\gamma=\alpha_{1}(+) \beta_{1}, \alpha_{1} \leq \alpha, \beta_{1} \leq \beta$. So $S_{\alpha}^{\omega}(X) \times S_{\beta}^{\omega}(X)=$ $\{*\} \cup(+)\left\{\left(S_{\nu}^{\omega}(X)\right)_{i}: \nu<\alpha(+) \beta, i<\omega\right\}$, where $\left(S_{\nu}^{\omega}(X)\right)_{i} \simeq S_{\nu}^{\omega}(X)$, $i<\omega$. By Definitions 1, 2 one finally has $S_{\alpha}^{\omega}(X) \times S_{\beta}^{\omega}(X)=S_{\alpha(+) \beta}^{\omega}(X)$. The theorem is proved.

Question 1. Can the assumption $\alpha, \beta<\omega_{1}$ be omitted in Theorem 1?

Re mark 1. $S_{\omega}(I) \times S_{\omega}(I) \not 千 S_{\omega(\cdot) 2}(I)$, because in $S_{\omega}(I) \times S_{\omega}(I)$ there is one isolated point and in $S_{\omega(\cdot) 2}(I)$ there are two isolated points.

Note, however, that for the ordinal powers there is a relation very close to equality:

Corollary 1. Let $\alpha, \beta<\omega_{1}$. Then

(a) $S_{\alpha(+) \beta}(X) \hookrightarrow S_{\alpha}(X) \times S_{\beta}(X) \hookrightarrow S_{\alpha(+) \beta}(X)$;

(b) $S_{\alpha}^{(\omega)}(X \times Y) \hookrightarrow S_{\alpha}^{(\omega)}(X) \times S_{\alpha}^{(\omega)}(Y) \hookrightarrow S_{p(\alpha)(\cdot) 2+n(\alpha)}^{(\omega)}(X \times Y)$.

P r o o f. (a) follows directly from Theorem 1 by using Lemma 1(b). Let us prove (b). We shall examine the case of ordinal products and use induction. 
Let $\alpha<\omega$. It is clear that $S_{\alpha}(X \times Y)=S_{\alpha}(X) \times S_{\alpha}(Y)$. Moreover, $p(\alpha)(\cdot) 2+n(\alpha)=\alpha$.

Let us prove the embedding $S_{\alpha}(X \times Y) \hookrightarrow S_{\alpha}(X) \times S_{\alpha}(Y)$ for $\alpha \geq \omega$. Assume that for $\alpha<\nu \geq \omega$ the statement is true, and let $\alpha=\nu$. Suppose that $\alpha=\varepsilon+1$. By the definition and the inductive assumption one easily has

$$
\begin{aligned}
S_{\alpha}(X) \times S_{\alpha}(Y) & =S_{\varepsilon}(X) \times S_{\varepsilon}(Y) \times X \times Y \\
& \hookleftarrow S_{\varepsilon}(X \times Y) \times(X \times Y)=S_{\alpha}(X \times Y) .
\end{aligned}
$$

Let now $\alpha$ be a limit ordinal. Then there exists an increasing sequence $\left\{\alpha_{i}\right\}_{i=1}^{\infty}$ of ordinals such that $\sup _{i} \alpha_{i}=\alpha$. Consider the chain of embeddings:

$$
\begin{aligned}
& S_{\alpha}(X) \times S_{\alpha}(Y) \\
& \left.\hookleftarrow\left(\left\{*_{1}\right\} \cup \underset{i=1}{\cup} \underset{+}{+}\right) S_{\alpha_{i}}(X)\right) \times\left(\left\{*_{2}\right\} \cup \underset{i=1}{\cup} \stackrel{\infty}{+} S_{\alpha_{i}}(Y)\right) \quad \text { (by Lemma 1) } \\
& \hookleftarrow\{*\} \cup \underset{i=1}{\stackrel{\infty}{+})} S_{\alpha_{i}}(X) \times S_{\alpha_{i}}(Y) \\
& \hookleftarrow\{*\} \cup \underset{i=1}{\infty} \stackrel{\infty}{+} S_{\alpha_{i}}(X \times Y) \quad \text { (by the inductive assumption) } \\
& \hookleftarrow S_{\alpha}(X \times Y) \quad \text { (by Lemma 1). }
\end{aligned}
$$

Let us prove the inverse embedding

$$
S_{\alpha}(X) \times S_{\alpha}(Y) \hookrightarrow S_{p(\alpha)(\cdot) 2+n(\alpha)}(X \times Y) .
$$

Assume that for $\alpha<\nu \geq \omega$ the statement is true, and let $\alpha=\nu$. Suppose $\alpha=\varepsilon+1$. Clearly,

$S_{\alpha}(X) \times S_{\alpha}(Y) \hookrightarrow S_{p(\varepsilon)(\cdot) 2+n(\varepsilon)}(X \times Y) \times(X \times Y)=S_{p(\alpha)(\cdot) 2+n(\alpha)}(X \times Y)$.

Suppose now that $\alpha$ is a limit ordinal. Then $p(\alpha)(\cdot) 2+n(\alpha)=\alpha(\cdot) 2$. Clearly,

$$
\begin{aligned}
S_{\alpha}(X) \times S_{\alpha}(Y) & \hookrightarrow S_{\alpha}(X \times Y) \times S_{\alpha}(X \times Y) \\
& \hookrightarrow S_{\alpha(+) \alpha}(X \times Y) \quad(\text { by }(\mathrm{a})) \\
& =S_{\alpha(\cdot) 2}(X \times Y) .
\end{aligned}
$$

The corollary is proved.

Now we get the following statement.

Corollary 2. Let $\Phi$ be a numerical function on topological spaces, monotone on closed sets, for example a dimension (ind, Ind, $\operatorname{dim}_{\mathrm{w}}, D$ or others). Then

$$
\Phi\left(S_{\alpha}^{(\omega)}(X) \times S_{\beta}^{(\omega)}(X)\right)=\Phi\left(S_{\alpha(+) \beta}^{(\omega)}(X)\right)
$$


In particular, for Smirnov compacta $S^{\gamma}, \gamma<\omega_{1}$, one has

(a) DIM $S^{\alpha} \times S^{\beta}=\alpha(+) \beta$, where DIM is $\operatorname{dim}_{\mathrm{w}}$, Ind, Id or $D$;

(b) ind $S^{\alpha} \times S^{\beta}=$ ind $S^{\alpha(+) \beta}$;

(c) $S^{\alpha} \times S^{\beta}$ can be continuously mapped into I so that every point of I has a finite-dimensional preimage.

Pr o of. Recall (see the introduction) that $\operatorname{dim}_{\mathrm{w}} S^{\alpha}=\operatorname{Ind} S^{\alpha}=D S^{\alpha}=$ Id $S^{\alpha}=\alpha$ for $\alpha<\omega_{1}$ (for Id see Corollary 5 in $\S 3$ ).

R e m a r k 2. Since the product $H^{\alpha} \times H^{\beta}$ cannot be continuously mapped into the interval with every point preimage finite-dimensional, and $H^{\gamma}$ can be mapped in such a way, the inclusion $H^{\alpha} \times H^{\beta} \hookrightarrow H^{\gamma}$ is not true for any infinite $\alpha, \beta, \gamma<\omega_{1}$. Therefore for the upper estimate of the dimension of $H^{\alpha} \times H^{\beta}$ we need Corollary 5 of $\S 3$, namely: $\operatorname{Id} H^{\alpha} \times H^{\beta} \leq \operatorname{Id} H^{\alpha}(+) \operatorname{Id} H^{\beta}=$ $\alpha(+) \beta$.

Since $S^{\alpha(+) \beta} \hookrightarrow S^{\alpha} \times S^{\beta} \hookrightarrow H^{\alpha} \times H^{\beta}$, the lower estimate is immediate; recall that $\operatorname{dim} X \leq \operatorname{Ind} X \leq \operatorname{Id} X$ for every compactum $X$. In the case of the dimension $D$, we need the following statements mentioned in the introduction: $D S^{\alpha}=\alpha, \alpha<\omega_{1}, D\left(H^{\alpha} \times H^{\beta}\right) \leq D H^{\alpha}(+) D H^{\beta}$ and the equalities $D H^{\gamma}=\gamma, \gamma<\omega_{1}$, which one easily checks by induction using the following properties of $D$ in the class of metrizable spaces [He2]:

- if either Ind $X$ or $D X$ is finite then they are equal;

- $D(X \times Y) \leq D X(+) D Y$;

- if $X$ is the union of a locally finite collection of closed subsets each with $D$-dimension $\leq \beta$, then $D X \leq \beta$;

- if $F$ is a closed subset of the space $X$ then $D X \leq D(X \backslash F)+D F$.

(For the definition of Henderson's compacta $H^{\gamma}, \gamma<\omega_{1}$, see the proof of Corollary 5.)

One finally has DIM $H^{\alpha} \times H^{\beta}=\alpha(+) \beta$, where DIM is Ind, Id, $\operatorname{dim}_{\mathrm{w}}$ or $D$, and $\alpha, \beta<\omega_{1}$.

In order to simplify formulas we write $\prod_{\gamma<\beta}^{\text {ord }} X_{\gamma}$ and $S_{\beta}(X)$ for ordinal $\aleph_{0}$-products and ordinal $\aleph_{0}$-powers. The notation $(+)_{\gamma<\beta} \alpha_{\gamma}$ denotes the inductive extension of the natural sum to infinite sequences of ordinals (see appendix).

TheOREM 2. Let $X$ be an arbitrary topological space and let ordinals $\beta$ and $\alpha_{\gamma} \geq 1, \gamma<\beta$, be countable. Then

$$
S_{(+)_{\gamma<\beta} \alpha_{\gamma}}(X) \hookrightarrow \prod_{\gamma<\beta}^{\text {ord }} S_{\alpha_{\gamma}}(X) \hookrightarrow S_{(+)_{\gamma<\beta} \alpha_{\gamma}}(X) .
$$

Proof. We use induction. Let $\beta<\omega$. Then $(*)$ is obvious by Corollary 1 . Suppose that the statement is true for all $\beta<\nu \geq \omega$, and let $\beta=\nu \geq \omega$. 
Assume that $\beta=\varepsilon+1$. Then by the inductive assumption one has

$$
S_{(+)_{\gamma<\varepsilon} \alpha_{\gamma}}(X) \hookrightarrow \prod_{\gamma<\varepsilon}^{\text {ord }} S_{\alpha_{\gamma}}(X) \hookrightarrow S_{(+)_{\gamma<\varepsilon} \alpha_{\gamma}}(X) .
$$

Multiply $(* *)$ by $S_{\alpha_{\varepsilon}}(X)$. By the definition one has $\left((+)_{\gamma<\varepsilon} \alpha_{\gamma}\right)(+) \alpha_{\varepsilon}=$ $(+)_{\gamma<\beta} \alpha_{\gamma}$. Now we only need to use either Corollary 1 or Theorem 1 .

Let now $\beta$ be a limit ordinal. Then there exists a sequence $\left\{\beta_{i}\right\}_{i=1}^{\infty}$ of ordinals such that $\sup _{i} \beta_{i}=\beta$. Set $\sigma_{i}=(+)_{\gamma<\beta_{i}} \alpha_{\gamma}$. It is clear that $(+)_{\gamma<\beta} \alpha_{\gamma}=\sup _{i} \sigma_{i}$. By the inductive assumption for every $i<\omega$ one has

$$
S_{\sigma_{i}}(X) \hookrightarrow \prod_{\gamma<\beta_{i}}^{\text {ord }} S_{\alpha_{\gamma}}(X) \hookrightarrow S_{\sigma_{i}}(X) .
$$

Moreover, obviously

$$
\prod_{\gamma<\beta}^{\text {ord }} S_{\alpha_{\gamma}}(X) \subset\{*\} \cup \underset{i=1}{\infty} \stackrel{\infty}{+} \underset{\gamma<\beta_{i}}{\text { ord }} S_{\alpha_{\gamma}}(X) \hookrightarrow \prod_{\gamma<\beta}^{\text {ord }} S_{\alpha_{\gamma}}(X)
$$

and

$$
S_{(+)_{\gamma<\beta} \alpha_{\gamma}}(X) \hookrightarrow\{*\} \cup \underset{i=1}{\stackrel{\infty}{\infty})} S_{\sigma_{i}}(X) \hookrightarrow S_{(+)_{\gamma<\beta} \alpha_{\gamma}}(X) .
$$

Our statement follows from these three chains of embeddings. The theorem is proved.

Remark 3. $S_{\omega}^{\omega}\left(S_{2}^{\omega}(I)\right) \neq S_{\omega}^{\omega}(I)$, though $2 \times \omega=\omega$.

Corollary 3. Let $\alpha, \beta<\omega_{1}$. Then

$$
S_{\alpha \times \beta}(X) \hookrightarrow S_{\beta}\left(S_{\alpha}(X)\right) \hookrightarrow S_{\alpha \times \beta}(X) .
$$

Proof. Let us only note that if we set $\alpha_{\gamma}=\alpha$ for all $\gamma<\beta$, then by the definition from the appendix we have $\alpha \times \beta=(+)_{\gamma<\beta} \alpha_{\gamma}$, and by the definition of the power $\prod_{\gamma<\beta}^{\text {ord }} S_{\alpha_{\gamma}}(X)=S_{\beta}\left(S_{\alpha}(X)\right)$.

Corollary 4. Let $\Phi$ be a numerical function on topological spaces, monotone on closed sets, for example a dimension (ind, Ind, $\operatorname{dim}_{\mathrm{w}}, D$ or others), and let $\alpha_{\gamma}, \beta<\omega_{1}$. Then

$$
\Phi\left(\prod_{\gamma<\beta}^{\text {ord }} S_{\alpha_{\gamma}}(X)\right)=\Phi\left(S_{(+)_{\gamma<\beta} \alpha_{\gamma}}(X)\right) .
$$

Note that the product $\prod_{\gamma<\beta}^{\text {ord }} S_{\alpha_{\gamma}}(I)$ can be continuously mapped into an interval with all point preimages finite-dimensional. For the product $\prod_{\gamma<\beta}^{\text {ord }} H_{\alpha_{\gamma}}(I)$ there is no such mapping.

3. Solution of the product problem for the inductive dimension functions id and $\mathrm{id}_{\mathrm{p}}$. Let $X$ be a normal space. 
Definition 3. Let $\operatorname{Id} X=-1$ iff $X=\emptyset$. Let $\operatorname{Id} X \leq \alpha$, where $\alpha$ is an ordinal, if there are collections $\sigma_{-1}, \sigma_{0}, \ldots, \sigma_{\delta}, \delta \leq \alpha$, of closed subsets of $X$ such that

(a) $\sigma_{-1}=\{\emptyset\}, \sigma_{\delta} \ni X, \sigma_{\beta} \subseteq \sigma_{\gamma}$ for all $\beta, \gamma$ with $-1 \leq \beta \leq \gamma \leq \delta$;

(b) for all $0 \leq \gamma \leq \delta$ and $F \in \sigma_{\gamma}$ and every pair of disjoint closed subsets $A$ and $B$ of $X$ there exist $\beta<\gamma$ and $\psi \in \sigma_{\beta}$ such that $\psi \subset F$ and $\psi$ is a partition in $F$ between $A \cap F$ and $B \cap F$;

(c) for every $\gamma \leq \delta$ and every pair $F_{1}, F_{2}$ of elements of $\sigma_{\gamma}$ there exists $F \in \sigma_{\gamma}$ such that $F \hookleftarrow F_{1} \cup F_{2}$.

Let us put $\operatorname{Id} X=\min \{\alpha: \operatorname{Id} X \leq \alpha\}$.

If Id $X \leq \alpha$ for no ordinal $\alpha$, then we define Id $X=\infty$ (which means that $\operatorname{Id} X$ does not exist).

Note that for every $F \in \sigma_{\gamma}, \gamma \leq \delta$, we have $\operatorname{Id} F \leq \gamma$.

If one of the sets from Definition 3, say $A$, is a single point, we get the definition of the dimension id.

If both $A, B$ are singletons, we get the definition of the dimension $\operatorname{id}_{\mathrm{p}}$. If both $A, B$ are compacta we get the definition of the dimension cId.

Clearly, $\operatorname{id}_{\mathrm{p}} X \leq \operatorname{id} X \leq \operatorname{Id} X$ and $\operatorname{id}_{\mathrm{p}} X \leq \operatorname{cId} X$ for every space $X$.

Remark 4. (a) The definitions of the finite dimensions id and Id and the statements given below for id and Id in the finite-dimensional case are due to Pasynkov [Pa1].

(b) The transfinite extensions of id and Id were independently considered by Vinogradov. He has independently proved the elementary properties of these dimensions given below, as well as Theorem 3 (for id) and Corollaries 5,6 .

Let DM be $\mathrm{id}_{\mathrm{p}}$, id, Id or cId, and DIM be ind $\mathrm{p}_{\mathrm{p}}$, ind, Ind or cInd (in the definition of ind $_{p}$ the partitions are taken between points and in the definition of cInd - between compacta [Ha]).

Statement 1. DIM $X \leq \alpha$ iff there exist collections $\sigma_{-1}, \ldots, \sigma_{\delta}, \delta \leq \alpha$, of closed subsets of $X$ satisfying conditions (a), (b) of Definition 3.

Proof. Let DIM $X=\delta \leq \alpha$. Put $\sigma_{\gamma}=\{Y \subseteq X: Y$ is closed in $X$ and DIM $Y \leq \gamma\}, \gamma=-1, \ldots, \delta$. It is clear that (a) and (b) are satisfied. Let us prove the converse. We use induction. Let $\alpha=-1$; then $X=\emptyset$ and, hence, DIM $X=-1$. Suppose that for $\alpha<\nu$ the statement is true, and let $\alpha=\nu$. By (a), $X \in \sigma_{\delta}, \delta \leq \nu$, and by (b) for every pair $A, B$ of disjoint closed subsets of $X$ there exist $\beta<\delta \leq \nu$ and $\psi \in \sigma_{\beta}$ such that $\psi$ is a partition in $X$ between $A$ and $B$. Consider the collections $\sigma_{\gamma}^{1}=\left\{C \cap \psi: C \in \sigma_{\gamma}\right\}$, $\gamma \leq \beta$, of subsets of $\psi$. It is clear that they satisfy conditions (a) and (b). By the inductive assumption we have $\operatorname{DIM} \psi \leq \beta<\nu$. Hence, DIM $X \leq \nu$. 
A collection $\mathcal{B}$ of closed subsets of $X$ is called monotone if every closed subset of any element of $\mathcal{B}$ also belongs to $\mathcal{B}$. A collection $\mathcal{B}$ of closed subsets of $X$ is called additive if for any $A, B \in \mathcal{B}$ we have $A \cup B \in \mathcal{B}$.

Statement 2. DM $X \leq \alpha$ iff there exist collections $\sigma_{-1}, \ldots, \sigma_{\delta}, \delta \leq \alpha$, of closed subsets of $X$ satisfying conditions (a), (b) of Definition 3 and the condition

$(\mathrm{c})^{1} \sigma_{\gamma}$ is monotone and additive for every $\gamma \leq \delta$.

Proof. The "if" part is clear. Let us prove the "only if" part. Let DM $X \leq \alpha$. Then there exist collections $\sigma_{-1}, \ldots, \sigma_{\delta}, \delta \leq \alpha$, of closed subsets of $X$ satisfying conditions (a), (b) and (c) of Definition 3. Put

$$
\sigma_{\gamma}^{1}=\left\{A \subseteq B: A \text { is closed in } X \text { and } B \in \sigma_{\gamma}\right\}, \quad \gamma \leq \delta .
$$

It is clear that these collections satisfy (a), (b) and (c) ${ }^{1}$.

Note that in Statement 2 one can always put $\delta=\alpha$. From Statements 1 and 2 we get

Statement 3. If DM $X$ exists, then DIM $X$ exists. Moreover, DIM $X \leq$ $\operatorname{DM} X$.

Statement 4. (i) For every compactum $X$ we have $\operatorname{id}_{\mathrm{p}} X=\operatorname{id} X=$ Id $X=\operatorname{cId} X$.

(ii) For every normal space $X$ we have $\operatorname{cId} X=\operatorname{id}_{\mathrm{p}} X$.

Proof. (i) Let $\operatorname{id}_{\mathrm{p}} X \leq \alpha$. There exist collections $\sigma_{-1}, \sigma_{0}, \ldots, \sigma_{\alpha}$ of closed subsets of $X$, satisfying (a), (b) and (c) ${ }^{1}$ (with $A$ and $B$ being points). By additivity and monotonicity (Statement 2 ), the same systems $\sigma_{\gamma}, \gamma \leq \alpha$, satisfy (a), (b) and (c) $)^{1}$ for $A$ and $B$ arbitrary closed disjoint sets. Thus (i) is proved. The proof of (ii) is analogous.

Statement 5. (i) If $F$ is closed in $X$, then $\mathrm{DM} F \leq \mathrm{DM} X$.

(ii) Suppose DIM $X$ exists and the finite sum theorem for DIM holds in $X$, for example, $X$ is a metric compactum with Ind $X \leq \omega$. Then $\operatorname{DM} X$ exists and $\operatorname{DM} X=\operatorname{DIM} X$.

(iii) Let $X_{\alpha}$ be a compactum with $\operatorname{DM} X_{\alpha}=\beta_{\alpha}, \alpha \in A,|A| \geq \omega$, and $\sup _{\alpha \in A} \beta_{\alpha}=\beta$. Let $X=\{*\} \cup(+)_{\alpha \in A} X_{\alpha}$ be the one-point Aleksandrov compactification of the free sum of the spaces $X_{\alpha}, \alpha \in A$. Then $\operatorname{DM} X$ exists and $\mathrm{DM} X=\beta$.

Proof. (i) is clear.

(ii) Let DIM $X=\alpha$. Then for $\gamma=-1,0, \ldots, \alpha$ we put

$$
\sigma_{\gamma}=\{Y \subseteq X: Y \text { is closed in } X \text { and } \operatorname{DIM} Y \leq \gamma\} .
$$

By the monotonicity of DIM on closed sets and the finite sum theorem one can easily check that the collections $\sigma_{\gamma}, \gamma \leq \alpha$, satisfy conditions (a), (b) 
and $(\mathrm{c})^{1}$. So $\operatorname{DM} X \leq \operatorname{DIM} X$. From Statement 3 one finally has $\operatorname{DM} X=$ $\operatorname{DIM} X$.

(iii) Let $\sigma_{\gamma}^{(\alpha)}, \gamma \leq \beta$, be collections of closed subsets of $X$ satisfying (a), (b) and (c) $)^{1}$, for $\alpha \in A$. Put

$$
\sigma_{\gamma}=\left\{A_{1} \cup \ldots \cup A_{k}: A_{i} \in \sigma_{\gamma}^{\left(\alpha_{i}\right)}, \alpha_{i} \in A, k \in \mathbb{N}\right\}, \quad \gamma<\beta,
$$

and $\sigma_{\beta}=\{A \subseteq X: A$ is compact $\}$. It is clear that for $\sigma_{-1}, \ldots, \sigma_{\beta}$ conditions (a), (b) and (c) $)^{1}$ are satisfied.

Hence, $\mathrm{DM} X \leq \beta$. Since $\mathrm{DM} X_{\alpha} \leq \mathrm{DM} X$ for every $\alpha \in A$, one finally obtains $\operatorname{DM} X=\beta$.

Questions. 2. Does DM $X$ exist if DIM $X$ exists?

3. It is known that for Smirnov compacta with Ind $>\omega$ the finite sum theorem for Ind does not hold [Le]. Does there exist a compactum $X$ with Ind $X>\omega$ in which the finite sum theorem for Ind holds?

4. By Filippov's $[\mathrm{F}]$ and Pasynkov's [Pa1] results there exists a compactum $X$ with Ind $X=2$ and $\operatorname{Id} X \geq 3$. How large may be the difference between Ind and Id for infinite-dimensional spaces (for DIM and DM)?

Lemma 2 (B. A. Pasynkov). Let $X=F_{1} \cup \ldots \cup F_{n}$ be a normal space, where $F_{i}, i=1, \ldots, n$, are closed in $X$. Let $A$ and $B$ be two disjoint closed subsets of $X$, and $C_{i}$ be a partition in $F_{i}$ between $A \cap F_{i}$ and $B \cap F_{i}, i=$ $1, \ldots, n$. Then there exists a partition $C$ in $X$ between $A$ and $B$ such that

$$
C \subseteq\left(\bigcup_{i=1}^{n} C_{i}\right) \cup \bigcup_{i<j}\left(F_{i} \cap F_{j}\right)
$$

The notation $\operatorname{id}_{(\mathrm{p})}$ will mean either $\mathrm{id}_{\mathrm{p}}$ or id. The main statement of this section is

Theorem 3. Let $X_{1} \times X_{2}$ be a normal space and suppose $\operatorname{id}_{(\mathrm{p})} X_{i}$ exists for $i=1,2$. Then

$$
\operatorname{id}_{(\mathrm{p})} X_{1} \times X_{2} \leq \operatorname{id}_{(\mathrm{p})} X_{1}(+) \operatorname{id}_{(\mathrm{p})} X_{2} .
$$

Proof. Let us show the inequality

$$
\operatorname{id}_{\mathrm{p}} X_{1} \times X_{2} \leq \operatorname{id}_{\mathrm{p}} X_{1}(+) \operatorname{id}_{\mathrm{p}} X_{2} .
$$

For id the proof of the corresponding inequality is analogous.

Let $\operatorname{id}_{\mathrm{p}} X_{1}=\xi, \operatorname{id}_{\mathrm{p}} X_{2}=\zeta$ and let $\sigma_{-1,1} \subseteq \ldots \subseteq \sigma_{\xi, 1}$ be collections of closed subsets of $X_{1}$, and $\sigma_{-1,2} \subseteq \ldots \subseteq \sigma_{\zeta, 2}$ collections of closed subsets of $X_{2}$ satisfying (a), (b) and (c) ${ }^{1}$ for $A$ and $B$ being points. Put

$$
\Sigma=\xi(+) \zeta, \quad \sigma_{-1}=\{\emptyset\}
$$




$$
\begin{aligned}
& \sigma_{\gamma}^{1}=\left\{A \times B: A \in \sigma_{\alpha, 1}, B \in \sigma_{\beta, 2} \text { and } \alpha(+) \beta \leq \gamma\right\}, \\
& \sigma_{\gamma}^{2}=\left\{C_{1} \cup \ldots \cup C_{k}: C_{i} \in \sigma_{\gamma}^{1}, i=1, \ldots, k, k \in \mathbb{N}\right\}, \\
& \sigma_{\gamma}=\left\{P \subseteq C: P \text { is closed in } X_{1} \times X_{2} \text { and } C \in \sigma_{\gamma}^{2}\right\}, \quad \gamma \leq \Sigma .
\end{aligned}
$$

Obviously, the collections $\sigma_{\gamma}, \gamma \leq \Sigma$, satisfy (a) and (c) $)^{1}$. Let us show that (b) holds. Let $P \in \sigma_{\gamma}$ and $A, B$ be a pair of different points in $P$. We have to show that there exist $\nu<\gamma$ and a set $C \in \sigma_{\nu}$ with $C \subseteq P$ which is a partition in $P$ between $A$ and $B$.

The main case. Let $P \subseteq D_{1} \times D_{2}$, where $D_{1} \in \sigma_{\alpha, 1}, D_{2} \in \sigma_{\beta, 2}$ and $\alpha(+) \beta \leq \gamma$. From the monotonicity of the collections $\sigma_{\gamma}, \gamma \leq \Sigma$, one can easily check that it is only necessary to consider the case $P=D_{1} \times D_{2}$.

Let $\pi: X_{1} \times X_{2} \rightarrow X_{1}$ be the projection. Since $A=B$, without loss of generality one can assume that $\pi A=\pi B$. There exists a partition $C_{1}$ in $D_{1}$ between the points $\pi A, \pi B$ such that $C_{1} \in \sigma_{\lambda, 1}, \lambda<\alpha$. Then clearly $\pi_{1}^{-1} C_{1}$ is a partition in $P$ between $A$ and $B$, and $\pi_{1}^{-1} C_{1} \in \sigma_{\lambda(+) \beta}$. Note that $\lambda(+) \beta<\alpha(+) \beta$ (see appendix).

The general case. Without loss of generality, by monotonicity and additivity of $\sigma_{\gamma}, \gamma \leq \Sigma$, and by Lemma 2 one can assume that $P=\left(D_{1}^{(1)} \times\right.$ $\left.D_{2}^{(1)}\right) \cup\left(D_{1}^{(2)} \times D_{2}^{(2)}\right)$, where $D_{1}^{(i)} \in \sigma_{\alpha_{i}, 1}, D_{2}^{(i)} \in \sigma_{\beta_{i}, 2}$ and $\alpha_{1}(+) \beta_{1} \leq \gamma$, $\alpha_{2}(+) \beta_{2} \leq \gamma$. Two cases are possible:

(I) $\alpha_{1}(+) \beta_{1}<\alpha_{2}(+) \beta_{2} \leq \gamma$. By the main case there exists a partition $C_{1}$ in $D_{1}^{(2)} \times D_{2}^{(2)}$ between $A$ and $B$ such that $C_{1} \in \sigma_{\mu}$ for some $\mu<\gamma$. By Lemma 2 one can choose a partition $C$ in $P$ between $A$ and $B$ such that $C \subseteq C_{1} \cup\left(D_{1}^{(1)} \times D_{2}^{(1)}\right)$. Let $\nu=\max \left(\alpha_{1}(+) \beta_{1}, \mu\right)<\gamma$. Then $C \in \sigma_{\nu}$.

(II) $\alpha_{1}(+) \beta_{1}=\alpha_{2}(+) \beta_{2}$. The following subcases are possible.

(II) $)_{1}$ Let $\alpha_{1}=\alpha_{2}=\alpha$. Then (see appendix) $\beta_{1}=\beta_{2}=\beta$. By additivity, $D_{1}=D_{1}^{(1)} \cup D_{1}^{(2)} \in \sigma_{\alpha, 1}, D_{2}=D_{2}^{(1)} \cup D_{2}^{(2)} \in \sigma_{\beta, 1}$ and $P \subseteq D_{1} \times D_{2}$. So the conditions of the main case are satisfied.

(II) $)_{2}$ Let $\alpha_{1}<\alpha_{2}$. Then (see appendix) $\beta_{1}>\beta_{2}$. In this case by monotonicity one has $D_{1}^{(1)} \cap D_{1}^{(2)} \in \sigma_{\alpha_{1}, 1}, D_{2}^{(1)} \cap D_{2}^{(2)} \in \sigma_{\beta_{2}, 2}$ and

$$
L=\left(D_{1}^{(1)} \times D_{2}^{(1)}\right) \cap\left(D_{1}^{(2)} \times D_{2}^{(2)}\right)=\left(D_{1}^{(1)} \cap D_{1}^{(2)}\right) \times\left(D_{2}^{(1)} \cap D_{2}^{(2)}\right) .
$$

Moreover, $\alpha_{1}(+) \beta_{2}<\alpha_{1}(+) \beta_{1}=\alpha_{2}(+) \beta_{2} \leq \gamma$ and $L \in \sigma_{\alpha_{1}(+) \beta_{2}}$. By the main case there exists a partition $C_{i}$ in $D_{1}^{(i)} \times D_{2}^{(i)}$ between $A$ and $B$ such that $C_{i} \in \sigma_{\mu_{i}}$ for some $\mu_{i}<\gamma, i=1,2$. By Lemma 1 there exists a partition $C$ in $P$ between $A$ and $B$ such that $C \subseteq L \cup C_{1} \cup C_{2}$. Let $\nu=\max \left(\alpha_{1}(+) \beta_{2}, \mu_{1}, \mu_{2}\right)<\gamma$. By the additivity and monotonicity of $\sigma_{\gamma}$, $\gamma \leq \Sigma$, we get $C \in \sigma_{\nu}$. The theorem is proved. 
In the sequel, dim stands for Borst's [B1] transfinite extension $\operatorname{dim}_{\mathrm{w}}$ of the Lebesgue covering dimension $\operatorname{dim}$. Recall that $\operatorname{dim} X \leq \operatorname{Ind} X$ for every normal space $X[\mathrm{~B} 1]$.

Corollary 5. (i) Let $X_{1}, X_{2}$ be compacta for which Id exists. Then

$$
\operatorname{dim} X_{1} \times X_{2} \leq \operatorname{Ind} X_{1} \times X_{2} \leq \operatorname{Id} X_{1} \times X_{2} \leq \operatorname{Id} X_{1}(+) \operatorname{Id} X_{2} .
$$

In particular, if $\operatorname{Id} X_{2}=n<\omega$, for example, $X_{2}=I^{n}$, then

$$
\operatorname{dim} X_{1} \times X_{2} \leq \operatorname{Ind} X_{1} \times X_{2} \leq \operatorname{Id} X_{1} \times X_{2} \leq \operatorname{Id} X_{1}+n .
$$

(ii) $\operatorname{Id} S^{\alpha}=\alpha$ for $\alpha<\omega_{1}$.

(iii) Id $H^{\alpha}=\alpha$ for $\alpha<\omega_{1}$.

Proof. (i) is evident.

(ii) One can easily check by induction using (i) and Statement 5(iii) that Id $S^{\alpha}=\alpha$ for $\alpha<\omega_{1}$ (recall that Ind $S^{\alpha}=\alpha$ for $\alpha<\omega_{1}$, see [S]).

(iii) Recall Henderson's description of $H^{\alpha}$. For $\alpha<\omega_{1}$, we define $H^{\alpha}$ and $p_{\alpha}$ as follows: $H^{0}=\{0\}, H^{1}=[0,1]=I$ and $p_{1}=0 ; H^{\alpha+1}=H^{\alpha} \times I$ and $p_{\alpha+1}=p_{\alpha} \times\{0\}$; if $\alpha$ is a limit ordinal, for $\beta<\alpha$ let $A_{\alpha}^{\beta}$ be a half-open arc with $H^{\beta} \cap A_{\alpha}^{\beta}=\left\{p_{\beta}\right\}$; then $H^{\alpha}=\{*\} \cup(+)_{\beta<\alpha}\left(H^{\beta} \cup A_{\alpha}^{\beta}\right)$ is the one point-compactification of the free sum where $p_{\alpha}=*$ is the compactification point. Since $S^{\alpha} \hookrightarrow H^{\alpha}$ and Id $S^{\alpha}=\alpha$ for $\alpha<\omega_{1}$ (see (ii)), we need to prove that Id $H^{\alpha} \leq \alpha$ for $\alpha<\omega_{1}$. If $\alpha$ is a non-limit ordinal we can use (i).

Let now $\alpha$ be a limit ordinal and suppose that Id $H^{\beta} \leq \beta$ for all $\beta<\alpha$. By Statement 4 , Id can be replaced by $\operatorname{id}_{\mathrm{p}}$ in the above inequality. Let $\sigma_{\gamma}^{(\beta)}$, $\gamma \leq \alpha$, be collections of closed subsets of $H^{\beta}$ satisfying (a), (b) and (c) $)^{1}$ (see Statement 2 for $\mathrm{id}_{\mathrm{p}}$ ) for $\beta<\alpha$. Put

$$
\begin{aligned}
& M=\{\emptyset\} \cup\left\{\text { finite subsets of } \bigcup\left\{A_{\alpha}^{\beta}: \beta<\alpha\right\}\right\}, \quad \sigma_{-1}=\{\emptyset\}, \\
& \sigma_{\gamma}=\left\{A_{1} \cup \ldots \cup A_{k} \cup P: A_{i} \in \sigma_{\gamma}^{\left(\beta_{i}\right)}, \beta_{i}<\alpha, k \in \mathbb{N}, P \in M\right\}, \quad \gamma<\alpha,
\end{aligned}
$$

and $\sigma_{\alpha}=\{A \subseteq X: A$ is compact $\}$. Obviously, $\sigma_{-1}, \ldots, \sigma_{\alpha}$ satisfy (a), (b) and $(\mathrm{c})^{1}$.

Corollary 6. Let $X_{1}$ and $X_{2}$ be compacta for which Ind exists and in which the finite sum theorem for Ind holds. Then

$$
\operatorname{dim} X_{1} \times X_{2} \leq \operatorname{Ind} X_{1} \times X_{2} \leq \operatorname{Ind} X_{1}(+) \operatorname{Ind} X_{2} .
$$

In particular, if Ind $X_{2}=n<\omega$, for example, $X_{2}=I^{n}$, then $\operatorname{dim} X_{1} \times X_{2} \leq$ Ind $X_{1} \times X_{2} \leq \operatorname{Ind} X_{1}+n$.

Question 5. Are there two metric compacta $X_{1}$ and $X_{2}$ such that Ind $X_{1} \times X_{2}>$ Ind $X_{1}(+)$ Ind $X_{2}$ ? The same question may be asked for $\operatorname{dim}_{\mathrm{w}}, \operatorname{dim}_{\mathrm{c}}$ and ind. 
Now let us recall the definition of metrizable spaces $M_{\alpha}, \alpha$ an ordinal, constructed by Hattori [Ha]. $M_{0}=\{*\}$ is a one-point space. Suppose that the metrizable spaces $M_{\beta}$ are defined for all $\beta<\alpha$. If $\alpha=\beta+1$, define $M_{\alpha}=M_{\beta} \times I$. If $\alpha$ is a limit ordinal, let $M_{\alpha}$ be the tolopogical sum of copies of all $M_{\beta}, \beta<\alpha$, together with a new point $x_{\alpha}$, with the following topology: for each $\beta<\alpha$, let $h_{\beta \alpha}$ be the natural embedding of $M_{\beta}$ in $M_{\alpha}$. Then $M_{\alpha}=\left\{x_{\alpha}\right\} \cup(+)\left\{h_{\beta \alpha}\left(M_{\beta}\right): \beta<\alpha\right\}$. A subset $U$ of $(+)\left\{h_{\beta \alpha}\left(M_{\beta}\right): \beta<\alpha\right\}$ is open in $M_{\alpha}$ if $U \cap h_{\beta \alpha}\left(M_{\beta}\right)$ is open in $h_{\beta \alpha}\left(M_{\beta}\right)\left(h_{\beta \alpha}\left(M_{\beta}\right)\right.$ is homeomorphic to $M_{\beta}$ ) for all $\beta<\alpha$. For every $n, m \in \mathbb{N}$ let

$$
\begin{aligned}
V_{m}(\alpha) & =(+)\left\{h_{\gamma+m, \alpha}\left(M_{\gamma+m}\right): \gamma<\alpha \text { is a limit ordinal }\right\}, \\
U_{n}\left(x_{\alpha}\right) & =\left\{x_{\alpha}\right\} \cup \bigcup\left\{V_{m}(\alpha): m \geq n\right\} .
\end{aligned}
$$

Let $\left\{U_{n}\left(x_{\alpha}\right): n \in \mathbb{N}\right\}$ be a base of neighborhoods at $x_{\alpha}$. This completes the inductive construction. In [Ha] Hattori showed the inequalities cInd $X \leq$ $\omega$ ind $X$ for every metric space $X$ and cInd $M_{\alpha} \geq \alpha$ for any $\alpha$, from which he got an affirmative answer to question 3.11 of [E3]: for every ordinal $\alpha$ there exists a metrizable space $X_{\alpha}$ with ind $X_{\alpha}=\alpha$. Note that Pasynkov [Pa2] had earlier stated the same result.

We have the following

Corollary 7. (i) $\operatorname{cId} M_{\alpha}=\mathrm{id}_{\mathrm{p}} M_{\alpha}=\alpha$;

(ii) $\operatorname{cId} M_{\alpha} \times M_{\beta}=\operatorname{id}_{\mathrm{p}} M_{\alpha} \times M_{\beta} \leq \alpha(+) \beta$.

Proof. Let us only note (see Statements 3 and 4 ) that $\alpha \leq \operatorname{cInd} M_{\alpha} \leq$ $\operatorname{cId} M_{\alpha}=\operatorname{id}_{\mathrm{p}} M_{\alpha}$ and $\operatorname{id}_{\mathrm{p}} I^{n}=n$.

4. Dimension of ordinal products. Let $\mathcal{B}=\left\{X_{\gamma}, \gamma<\beta\right\}$ be a $\beta$ sequence of topological spaces. In this section, $\prod_{\gamma<\beta}^{\text {ord }} X_{\gamma}$ stands for either $\prod_{\gamma<\beta}^{\text {ord }} X_{\gamma}$ or $\prod_{\gamma<\beta}^{\omega, \text { ord }} X_{\gamma}$.

TheOREM 4. Suppose $\prod_{\gamma<\beta}^{\text {ord }} X_{\gamma}$ is a normal space (for example, all $X_{\gamma}$, $\gamma<\beta$, are metric and $\left.\beta<\omega_{1}\right)$. Then

$$
\operatorname{id}_{(\mathrm{p})} \prod_{\gamma<\beta}^{\text {ord }} X_{\gamma} \leq \underset{\gamma<\beta}{(+) \operatorname{id}_{(\mathrm{p})}} X_{\gamma} .
$$

P r o o f. The statement directly follows from Theorem 3, Statement 5 and the definition of the natural sum of a $\beta$-sequence of ordinals (see appendix).

COROLlary 8. Let $X_{\gamma}, \gamma<\beta$, be compact spaces. Then

$$
\text { Id } \prod_{\gamma<\beta}^{\text {ord }} X_{\gamma} \leq \underset{\gamma<\beta}{(+)} \operatorname{Id} X_{\gamma} .
$$

Proof. Use Statement 4. 
Corollary 9. (i) Suppose $S_{\beta}(X)$ is a normal space and $\operatorname{id}_{(\mathrm{p})} X=\alpha$. Then $\operatorname{id}_{(\mathrm{p})} S_{\beta}(X) \leq \alpha \times \beta$.

(ii) ind $S_{\alpha}\left(\mathbb{R}^{k}\right) \leq \operatorname{id} S_{\alpha}\left(\mathbb{R}^{k}\right) \leq p(\alpha)+k \cdot n(\alpha)$, where $\mathbb{R}$ is the real line (note that ind $S_{\omega+3}(\mathbb{R})=\omega+2<\omega+3$, see [L]).

(iii) If $X_{\gamma}, \gamma<\beta$, are finite-dimensional metric compacta, then

$$
\operatorname{dim} \prod_{\gamma<\beta}^{\text {ord }} X_{\gamma} \leq \operatorname{Ind} \prod_{\gamma<\beta}^{\text {ord }} X_{\gamma} \leq \operatorname{Id} \prod_{\gamma<\beta}^{\text {ord }} X_{\gamma} \leq \underset{\gamma<\beta}{(+)} \operatorname{dim} X_{\gamma} .
$$

(iv) If all $X_{\gamma}, \gamma<\beta$, are finite-dimensional in the sense of $\operatorname{ind}_{(\mathrm{p})}$, the finite sum theorem is satisfied for this dimension and $\prod_{\gamma<\beta}^{\text {ord }} X_{\gamma}$ is a normal space, then

$$
\operatorname{ind}_{(\mathrm{p})} \prod_{\gamma<\beta}^{\text {ord }} X_{\gamma} \leq \operatorname{id}_{(\mathrm{p})} \prod_{\gamma<\beta}^{\text {ord }} X_{\gamma} \leq \underset{\gamma<\beta}{(+) \operatorname{ind}_{(\mathrm{p})}} X_{\gamma} .
$$

Proof. Just note that $\alpha \times \beta=(+)_{\gamma<\beta} \alpha_{\gamma}$ where $\alpha_{\gamma}=\alpha$ for all $\gamma$.

For $L$ an arbitrary set, Fin $L$ is the family of all finite non-empty subsets of $L$. The notation $L(X)$ stands for the family of all pairs of disjoint closed subsets of the topological space $X$. A finite set $\left\{\left(A_{i}, B_{i}\right)\right\}_{i=1}^{n} \in \operatorname{Fin} L(X)$ is called essential if every collection of partitions $\left\{C_{i}\right\}_{i=1}^{n}$, where $C_{i}$ is a partition between $A_{i}$ and $B_{i}$, has non-empty intersection. Put $M_{L}=\{\sigma \in$ Fin $L: \sigma$ is essential\} for $L \subseteq L(X)$.

Lemma 3 (A. N. Dranishnikov [D]). Let $\left\{\left(A_{i}, B_{i}\right)\right\}_{i=1}^{n}$ be an essential set in a metric compactum $X, Z$ a metric continuum and $z^{+}, z^{-}$different points in $Z$. Then the set $\left\{\left(A_{i} \times Z, B_{i} \times Z\right)\right\}_{i=1}^{n} \cup\left(X \times z^{+}, X \times z^{-}\right)$is essential in $X \times Z$.

Let $X_{\gamma}, \gamma<\beta$, be metric one-dimensional continua and $z_{\gamma}^{+}$, $z_{\gamma}^{-}$be different points in $X_{\gamma}$. For every $\delta \leq \beta$ we define a subset $P_{\delta}$ of $L\left(\prod_{\gamma<\delta}^{\text {ord }} X_{\gamma}\right)$ by

$$
\begin{aligned}
P_{1}= & \left\{\left(z_{0}^{-}, z_{0}^{+}\right)\right\}, \\
P_{\delta+1}= & \left\{\left(F \times X_{\delta}, G \times X_{\delta}\right):(F, G) \in P_{\delta}\right\} \\
& \cup\left(\prod_{\gamma<\delta}^{\text {ord }} X_{\gamma} \times z_{\delta}^{-}, \prod_{\gamma<\delta}^{\text {ord }} X_{\gamma} \times z_{\delta}^{+}\right),
\end{aligned}
$$

and if $\delta$ is a limit number, then

$$
P_{\delta}=\left\{\left(i_{\delta}^{\mu}(F), i_{\delta}^{\mu}(G)\right):(F, G) \in P_{\mu}, \mu<\delta\right\},
$$

where $i_{\delta}^{\mu}$ is the natural embedding of $\prod_{\gamma<\mu}^{\text {ord }} X_{\gamma}$ into $\prod_{\gamma<\delta}^{\text {ord }} X_{\gamma}$.

Using Lemma 3 one can show (see [B2]) that for $\alpha \leq \beta$,

$$
\operatorname{Ord} M_{P_{\alpha}} \geq \alpha
$$


where Ord is Borst's transfinite function. Note that for every $L \subseteq L(X)$ and every normal space $X$ the following inequalities are satisfied [B2]:

$$
\operatorname{Ord} M_{L} \leq \operatorname{Ord} M_{L(X)}=\operatorname{dim} X \leq \operatorname{Ind} X \text {. }
$$

Theorem 5. Let DIM be dim, Ind or Id, and let $X_{\gamma}, \gamma<\beta$, be onedimensional metric compacta. Then DIM $\prod_{\gamma<\beta}^{\text {ord }} X_{\gamma}=\beta$.

Proof. The estimate $\operatorname{Id} \prod_{\gamma<\beta}^{\text {ord }} X_{\gamma} \leq \beta$ follows from Corollary 8 . The rest follows from $(* *)$ and $(* * *)$.

Corollary 10. Let DIM be dim, Ind or Id, let $X$ be a one-dimensional metric compactum and let $\alpha_{\gamma}, \beta<\omega_{1}$. Then

$$
\operatorname{DIM} \prod_{\gamma<\beta}^{\text {ord }} S_{\alpha_{\gamma}}(X)=\underset{\gamma<\beta}{(+)} \alpha_{\gamma} .
$$

In particular, if, for each $\alpha<\omega_{1}, X^{\alpha}$ is the Smirnov compactum $S^{\alpha}$ or the Henderson compactum $H^{\alpha}$, then

$$
\operatorname{DIM} \prod_{\gamma<\beta}^{\text {ord }} X^{\alpha_{\gamma}}=\underset{\gamma<\beta}{(+)} \alpha_{\gamma} .
$$

Proof. By Corollary 4 we have

$$
\operatorname{DIM}\left(\prod_{\gamma<\beta}^{\text {ord }} S_{\alpha_{\gamma}}(X)\right)=\operatorname{DIM}\left(S_{(+)_{\gamma<\beta} \alpha_{\gamma}}(X)\right) .
$$

So by Theorem $5, \operatorname{DIM}\left(S_{(+)_{\gamma<\beta} \alpha_{\gamma}}(X)\right)=(+)_{\gamma<\beta} \alpha_{\gamma}$. Recall that $S_{\gamma}(I)=S^{\gamma}$, $\gamma<\omega_{1}$.

In the case of Henderson compacta we have

$$
\operatorname{Id} \prod_{\gamma<\beta}^{\text {ord }} H^{\alpha_{\gamma}} \leq \underset{\gamma<\beta}{(+)} \operatorname{Id} H^{\alpha_{\gamma}}
$$

(see Corollary 8),

$$
\underset{\gamma<\beta}{(+) \operatorname{Id}} H^{\alpha_{\gamma}}=\underset{\gamma<\beta}{(+)} \alpha_{\gamma}
$$

(recall that Id $H^{\alpha_{\gamma}}=\alpha_{\gamma}$, see Corollary 5), $\prod_{\gamma<\beta}^{\text {ord }} S^{\alpha_{\gamma}} \subseteq \prod_{\gamma<\beta}^{\text {ord }} H^{\alpha_{\gamma}}$, and the inequalities $\operatorname{dim} X \leq \operatorname{Ind} X \leq \operatorname{Id} X$ hold for every compactum $X$.

The author thanks the referee for his valuable remarks.

Appendix. Let us recall (see $[\mathrm{K}-\mathrm{M}]$ ) some notions and statements from set theory. For every ordinal $\alpha$ the power with base $\omega$ and exponent $\alpha$ is defined by $\omega^{0}=0, \omega^{\xi+1}=\omega^{\xi} \cdot \omega$, and $\omega^{\lambda}=\sup _{\gamma<\lambda} \omega^{\gamma}$ if $\lambda$ is a limit ordinal.

Some properties of the power:

- if $\alpha<\beta$, then $\omega^{\alpha}<\omega^{\beta}$;

- $\omega^{\xi+\eta}=\omega^{\xi} \cdot \omega^{\eta}$; 
- $\left(\omega^{\xi}\right)^{\eta}=\omega^{\xi \cdot \eta}$

- if $\eta>\xi_{1}>\ldots>\xi_{p}$ and $n_{i} \in \mathbb{N}, i=1, \ldots, p$, then

$$
\omega^{\eta}>\omega^{\xi_{1}} \cdot n_{1}+\ldots+\omega^{\xi_{p}} \cdot n_{p} ;
$$

- every ordinal $\alpha$ can be uniquely represented as

$$
\alpha=\omega^{\eta_{1}} \cdot n_{1}+\ldots+\omega^{\eta_{k}} \cdot n_{k}, \quad n_{i} \in \mathbb{N},
$$

where $\eta_{1}>\ldots>\eta_{k} \geq 0$ are ordinals.

Let $\alpha$ and $\beta$ be ordinals and

$$
\alpha=\omega^{\eta_{1}} \cdot n_{1}+\ldots+\omega^{\eta_{k}} \cdot n_{k}, \quad \beta=\omega^{\zeta_{1}} \cdot m_{1}+\ldots+\omega^{\zeta_{l}} \cdot m_{l} .
$$

Adding powers with zero coefficients we get expansions with the same powers of $\omega$ :

(*) $\quad \alpha=\omega^{\xi_{1}} \cdot p_{1}+\ldots+\omega^{\xi_{h}} \cdot p_{h}, \quad \beta=\omega^{\xi_{1}} \cdot q_{1}+\ldots+\omega^{\xi_{h}} \cdot q_{h}$.

The ordinal

$$
\alpha(+) \beta=\omega^{\xi_{1}} \cdot\left(p_{1}+q_{1}\right)+\ldots+\omega^{\xi_{h}} \cdot\left(p_{h}+q_{h}\right)
$$

is called the natural sum of $\alpha$ and $\beta$. Their natural product $\alpha(\cdot) \beta$ is obtained by multiplying the expansions $(*)$ as polynomials in $\omega$, i.e. multiplying two powers of $\omega$ we take the natural sum of the exponents and arrange the monomials in the decreasing order of exponents.

Let $\Phi(\alpha, \beta)$ denote either $\alpha(+) \beta$ or $\alpha(\cdot) \beta$. Then

- $\Phi(\alpha, \beta)=\Phi(\beta, \alpha)$;

- if $\alpha_{1}<\alpha_{2}$, then $\Phi\left(\alpha_{1}, \beta\right)<\Phi\left(\alpha_{2}, \beta\right)$;

- if $\Phi\left(\alpha_{1}, \beta_{1}\right)=\Phi\left(\alpha_{2}, \beta_{2}\right)$, then $\alpha_{1}=\alpha_{2}$ implies $\beta_{1}=\beta_{2}$, and $\alpha_{1}<\alpha_{2}$ implies $\beta_{1}>\beta_{2}$;

- $\alpha(+) \ldots(+) \alpha(n$ times $)=\alpha(\cdot) n$ for $n<\omega$;

- $\alpha(+) n=\alpha+n$ for $n<\omega$.

A function $\varphi$ defined on a completely ordered set $W(\alpha)=\{\gamma: \gamma<\alpha\}$ of type $\alpha$ is called a transfinite sequence of type $\alpha$ or an $\alpha$-sequence. If the values of this sequence are ordinals and if $\gamma<\beta<\alpha$ implies $\varphi(\gamma)<\varphi(\beta)$ then this sequence is called increasing.

Let $\lambda$ be a limit ordinal and $\varphi$ be an increasing $\lambda$-sequence. Then

$$
\begin{aligned}
\sup _{\xi<\lambda}(\alpha+\varphi(\xi))= & \alpha+\sup _{\xi<\lambda} \varphi(\xi), \quad \sup _{\xi<\lambda}(\alpha \cdot \varphi(\xi))=\alpha \cdot \sup _{\xi<\lambda} \varphi(\xi), \\
& \sup _{\xi<\lambda} \omega^{\alpha \cdot \varphi(\xi)}=\omega^{\alpha \cdot \sup _{\xi<\lambda} \varphi(\xi)} .
\end{aligned}
$$

Lemma A1 $[\mathrm{H}]$. Let $\alpha, \beta<\omega_{1}$. Then for every $\gamma<\alpha(+) \beta$ there exist finitely many pairs $\alpha_{1}, \beta_{1}$ such that $\gamma=\alpha_{1}(+) \beta_{1}$ and $\alpha_{1} \leq \alpha, \beta_{1} \leq \beta$. 
ExAmPle A1. Let $\alpha=\beta=\omega^{2}$. Then

$$
\alpha(+) \beta=\omega^{2} \cdot 2 \neq \sup _{n, m}(\omega \cdot n(+) \omega \cdot m)=\omega^{2} .
$$

Let $\mathcal{B}=\left\{\alpha_{\gamma}, \gamma<\beta\right\}$ be a $\beta$-sequence of ordinals. The sum of all numbers of $\mathcal{B}$ is defined by

$$
\sum_{\gamma<\beta} \alpha_{\gamma}= \begin{cases}\alpha_{0} & \text { if } \beta=1 \\ \left(\sum_{\gamma<\delta} \alpha_{\gamma}\right)+\alpha_{\delta} & \text { if } \beta=\delta+1 \\ \sup _{\delta<\beta}\left(\sum_{\gamma<\delta} \alpha_{\gamma}\right) & \text { if } \beta \text { is a limit ordinal }\end{cases}
$$

(see, for example, $[\mathrm{H}]$ ).

The natural sum of all numbers of $\mathcal{B}$ is the number

$$
\underset{\gamma<\beta}{(+)} \alpha_{\gamma}= \begin{cases}\alpha_{0} & \text { if } \beta=1 \\ \left((+)_{\gamma<\delta} \alpha_{\gamma}\right)(+) \alpha_{\delta} & \text { if } \beta=\delta+1 \\ \sup _{\delta<\beta}(+)_{\gamma<\delta} \alpha_{\gamma} & \text { if } \beta \text { is a limit ordinal. }\end{cases}
$$

Clearly, if $\gamma<\delta<\beta$, then $\sum_{\mu<\gamma} \alpha_{\mu} \leq \sum_{\mu<\delta} \alpha_{\mu}$ and $(+)_{\mu<\gamma} \alpha_{\mu} \leq$ $(+)_{\mu<\delta} \alpha_{\mu}$, moreover, $\sum_{\gamma<\beta} \alpha_{\gamma} \leq(+)_{\gamma<\beta} \alpha_{\gamma}$.

It is well known that every ordinal $\alpha$ may be represented in the form $\alpha=p(\alpha)+n(\alpha)$, where $p(\alpha)$ is a limit ordinal ( 0 is considered to be a limit ordinal) and $n(\alpha)<\omega$.

One can easily check the following properties:

- If all $\alpha_{\gamma}, \gamma<\beta$, are equal to 1 , then $\sum_{\gamma<\beta} \alpha_{\gamma}=(+)_{\gamma<\beta} \alpha_{\gamma}=\beta$.

- If $1 \leq \alpha_{\gamma}<\omega$ for $\gamma<\beta$, then

$$
\sum_{\gamma<\beta} \alpha_{\gamma}=\underset{\gamma<\beta}{(+)} \alpha_{\gamma}= \begin{cases}p(\beta)+\alpha_{p(\beta)}+\ldots+\alpha_{\beta-1} & \text { if } \beta \text { is a non-limit ordinal, } \\ \beta & \text { if } \beta \text { is a limit ordinal. }\end{cases}
$$

- $\sum_{\gamma<\beta} \min _{\gamma<\beta} \alpha_{\gamma} \leq \sum_{\gamma<\beta} \alpha_{\gamma} \leq \sum_{\gamma<\beta} \sup _{\gamma<\beta} \alpha_{\gamma}$,

$$
\underset{\gamma<\beta}{(+)} \min _{\gamma<\beta} \alpha_{\gamma} \leq \underset{\gamma<\beta}{(+)} \alpha_{\gamma} \leq \underset{\gamma<\beta \gamma<\beta}{(+)} \sup _{\gamma} \alpha_{\gamma} \text {. }
$$

- $\sum_{\gamma<\beta} \alpha_{\gamma}=\alpha \cdot \beta$ if all $\alpha_{\gamma}=\alpha, \gamma<\beta$.

In view of the last equality, it is natural to give another definition of the product of two ordinals $\alpha$ and $\beta$ :

$$
\alpha \times \beta=\underset{\gamma<\beta}{(+)} \alpha_{\gamma},
$$

where $\alpha_{\gamma}=\alpha$ for $\gamma<\beta$. It is clear that $\alpha \cdot \beta \leq \alpha \times \beta$ and there exist pairs $\alpha, \beta$ such that $\alpha \cdot \beta<\alpha \times \beta$, for example,

$$
(\omega+1) \cdot 2=\omega \cdot 2+1<(\omega+1) \times 2=\omega \cdot 2+2 .
$$


It is not difficult to establish a relation between the new product and the usual operations on ordinals. We indicate it without proof:

Let $\alpha=\omega^{\eta_{1}} \cdot n_{1}+\ldots+\omega^{\eta_{k}} \cdot n_{k}, n_{i} \in \mathbb{N}$, and $\eta_{1}>\ldots>\eta_{k}$. Then

$$
\alpha \times \beta=\omega^{\eta_{1}} \cdot p(\beta)+\alpha(\cdot) n(\beta) .
$$

Note that $\alpha \times \beta \leq \alpha(\cdot) \beta$ and there exist pairs $\alpha, \beta$ such that $\alpha \times \beta<\alpha(\cdot) \beta$, for example $2 \times \omega=\omega<2(\cdot) \omega=\omega \cdot 2$. Note also that

- if $\beta_{1}<\beta_{2}$, then $\alpha \times \beta_{1}<\alpha \times \beta_{2}, \alpha>0$;

- $\alpha \times\left(\beta_{1}(+) \beta_{2}\right)=\left(\alpha \times \beta_{1}\right)(+)\left(\alpha \times \beta_{2}\right)$;

- if $\lambda$ is a limit ordinal and $\varphi$ is an increasing $\lambda$-sequence, then $\sup _{\xi<\lambda}(\alpha \times \varphi(\xi))=\alpha \times \sup _{\xi<\lambda} \varphi(\xi)$.

For the new product one may also introduce the notion of power.

\section{References}

[A-Pa] P. S. Aleksandrov and B. A. Pasynkov, Introduction to Dimension Theory, Nauka, Moscow, 1973 (in Russian).

[B1] P. Borst, Classification of weakly infinite-dimensional spaces. Part I: A transfinite extension of the covering dimension, Fund. Math. 130 (1988), 1-25.

[B2] -, Classification of weakly infinite-dimensional spaces. Part II: Essential mappings, ibid., 73-99.

[B3] - Some remarks concerning $C$-spaces, preprint.

[D] A. N. Dranishnikov, Absolute extensors in dimension $n$ and dimension raising n-soft mappings, Uspekhi Mat. Nauk 39 (5) (1984), 55-95 (in Russian).

[E1] R. Engelking, General Topology, PWN, Warszawa 1977.

[E2] -, Dimension Theory, PWN, Warszawa 1978.

[E3] -, Transfinite dimension, in: Surveys in General Topology, G. M. Reed (ed.), Academic Press, New York, 1980, 131-161.

[F] V. V. Filippov, On the inductive dimension of the product of bicompacta, Dokl. Akad. Nauk SSSR 202 (1972), 1016-1019 (in Russian).

[Ha] Y. Hattori, Solution of problems concerning transfinite dimension, Questions Answers Gen. Topology 1 (1983), 128-134.

[Ha-Y] Y. Hattori and K. Yamada, Closed pre-images of C-spaces, Math. Japon. 34 (1989), 555-561.

[H] F. Hausdorff, Set Theory, Chelsea, New York, 1962.

[He1] D. W. Henderson, A lower bound for transfinite dimension, Fund. Math. 63 (1968), 167-173.

[He2] -, D-dimension I. A new transfinite dimension, Pacific J. Math. 26 (1968), 91-107.

[Hes] G. Hessenberg, Grundbegriffe der Mengenlehre, Göttingen, 1906.

[K-M] K. Kuratowski and A. Mostowski, Set Theory, PWN and North-Holland, 1976.

[Le] B. T. Levshenko, Spaces of transfinite dimensionality, Mat. Sb. 67 (1965), 255-266 (in Russian); English transl.: Amer. Math. Soc. Transl. (2) 73 (1968), $135-148$. 
[L] L. A. Luxemburg, On compacta with non-coinciding transfinite dimensions, Dokl. Akad. Nauk SSSR 212 (1973), 1297-1300 (in Russian); English transl.: Soviet Math. Dokl. 14 (1973), 1593-1597.

[Pa1] B. A. Pasynkov, On dimension of rectangular products, Dokl. Akad. Nauk SSSR 221 (1975), 291-294 (in Russian).

[Pa2] - On transfinite dimension, Abstracts of Leningrad Internat. Topology Conf., 1982 (in Russian).

[P] R. Pol, On classification of weakly infinite-dimensional compacta, Fund. Math. 116 (1983), 169-188.

[Po] L. Polkowski, On transfinite dimension, Colloq. Math. 50 (1985), 61-79.

[S] Yu. M. Smirnov, On universal spaces for some classes of infinite-dimensional spaces, Izv. Akad. Nauk SSSR 23 (1959), 185-196 (in Russian); English transl.: Amer. Math. Soc. Transl. (2) 21 (1962), 21-34.

[T] G. H. Toulmin, Shuffling ordinals and transfinite dimension, Proc. London Math. Soc. 4 (1954), 177-195.

SCIENTIFIC RESEARCH INSTITUTE OF SYSTEM ANALYSIS RUSSIAN ACADEMY OF SCIENCES

AVTOZAVODSKAYA 23

MOSCOW 109280, RUSSIA

Received 19 May 1992;

in revised form 9 February and 20 May 1993 